\title{
Molecular mechanisms of fat deposition: IL-6 is a hub gene in fat lipolysis, comparing thin-tailed with fat-tailed sheep breeds
}

\author{
Sana Farhadi ${ }^{1}$, Jalil Shodja Ghias ${ }^{1}$, Karim Hasanpur ${ }^{1}$, Seyed Abolghasem Mohammadi $^{1}$, and \\ Esmaeil Ebrahimie ${ }^{2,3,4}$ \\ ${ }^{1}$ Department of Animal Science, Faculty of Agriculture, University of Tabriz, Tabriz, Iran \\ ${ }^{2}$ School of Animal and Veterinary Sciences, The University of Adelaide, South Australia 5371, Australia \\ ${ }^{3}$ School of BioSciences, The University of Melbourne, Melbourne, Australia \\ ${ }^{4}$ Genomics Research Platform, School of Life Sciences, \\ La Trobe University, Melbourne, Victoria 3086, Australia
}

Correspondence: Sana Farhadi (farhadiso16@tabrizu.ac.ir)

Received: 7 October 2020 - Revised: 14 December 2020 - Accepted: 18 December 2020 - Published: 17 February 2021

\begin{abstract}
Tail fat content affects meat quality and varies significantly among different breeds of sheep. Ghezel (fat-tailed) and Zel (thin-tailed) are two important Iranian local sheep breeds with different patterns of fat storage. The current study presents the transcriptome characterization of tail fat using RNA sequencing in order to get a better comprehension of the molecular mechanism of lipid storage in the two mentioned sheep breeds. Seven $(\mathrm{Zel}=4$ and Ghezel $=3) 7$-month-old male lambs were used for this experiment. The results of sequencing were analyzed with bioinformatics methods, including differentially expressed genes (DEGs) identification, functional enrichment analysis, structural classification of proteins, protein-protein interaction (PPI) and network and module analyses. Some of the DEGs, such as LIPG, SAAl, SOCS3, HIF- $1 \alpha$, and especially IL-6, had a close association with lipid metabolism. Furthermore, functional enrichment analysis revealed pathways associated with fat deposition, including "fatty acid metabolism", "fatty acid biosynthesis" and "HIF-l signaling pathway". The structural classification of proteins showed that major down-regulated DEGs in the Zel (thin-tailed) breed were classified under transporter class and that most of them belonged to the solute carrier transporter (SLC) families. In addition, DEGs under the transcription factor class with an important role in lipolysis were up-regulated in the Zel (thin-tailed) breed. Also, network analysis revealed that $I L-6$ and $J U N B$ were hub genes for up-regulated PPI networks, and HMGCS1, VPS35 and VPS26A were hub genes for down-regulated PPI networks. Among the up-regulated DEGs, the $I L-6$ gene seems to play an important role in lipolysis of tail fat in thin-tailed sheep breeds via various pathways such as tumor necrosis factor (TNF) signaling and mitogen-activated protein kinase (MAPK) signaling pathways. Due to the probable role of the $I L-6$ gene in fat lipolysis and also due to the strong interaction of $I L-6$ with the other up-regulated DEGs, it seems that $I L-6$ accelerates the degradation of lipids in tail fat cells.
\end{abstract}

\section{Introduction}

The development of adipose tissue has an effect on its function. Adipose tissue first appears around mid-gestation. Then, total adipose mass increases over the final few weeks of gestation (Symonds et al., 2012). In this time, it comprises a combination of brown and white adipocytes to en- able the newborn to effectively adapt to the thermal and nutritional challenges of life after birth. Then, during postnatal life some, but not all, storage is replaced by white adipose tissue (Symonds, 2013).

The formation of adipose tissue in humans occurs with the growth of small and larger adipocytes and slow storage of fat within the cell until the age of 6-12 months postnatally. 
Small cells in the early stages of fat storage have an important role in fat mass and lipid storage in the first 6-12 months of life and are associated with increased fat cell size (Boulton et al., 1978).

Sheep are the main livestock producers of meat, milk and wool in the world, with nearly $25 \%$ of the sheep population in the world being fat-tailed sheep (Zhou et al., 2017). The main factor influencing meat quality is the level of lipid storage in the carcass. Fat deposition efficiency in the tails of fat-tailed sheep is considerably higher than the other components of the carcass (Braissant et al., 1996; Li et al., 2018).

The most important component of the Iranian livestock industry is sheep production, which constitutes a total of nearly 50 million heads including more than 28 native sheep breeds which have great divergence in tail types (Moradi et al., 2012). Zel is the only thin-tailed breed (average $11 \mathrm{~cm}$ tail length), whereas the remaining breeds are considered fattailed (Valizadeh, 2010). The Zel (thin-tailed) breed is concentrated near the Caspian Sea, with almost $3 \%$ of the Iranian sheep population (Vatankhah et al., 2006; Kamalzadeh et al., 2008). The Ghezel, in contrast, is a fat-tailed breed in the northwest of Iran, which possesses a large fat tail (Nabavi et al., 2014).

The tail fat is considered as an adaptive response of sheep to a hazardous environment and is a rich energy reservoir for the animal in winter and during migration when feed is scarce (Atti et al., 2004). It was also traditionally utilized by human beings as a preserver to save cooked meat for a longer period of storage time and also as an energy-rich food resource. Therefore, climatic changes as well as the associated requirements of human beings led to selection for heavier fat-tailed sheep across many generations (Atti et al., 2004; Kashan et al., 2005; Ermias et al., 2002; Gokdal et al., 2004). In spite of the previous efforts and natural forces that have led to the creation of heavy, fat-tailed breeds, it is not considered as a good characteristic in modern sheep production. Now, it is believed that the heavier tail decreases feed efficiency and that more energy is needed to deposit fat in the tail than to produce an equivalent amount of other tissue. The emphasis is on channeling the nutrients into leaner carcass generation to diminish the costs of fat deposition (Moradi et al., 2012; Bolger et al., 2014). Also, the market demand and monetary value of the fat-tailed breeds have been reduced and the tail fat has lost much of its advantage (Moradi et al., 2012). Furthermore, excessive fat in human daily diets causes some health problems. Therefore, a smaller fat tail is now desirable for both producers and consumers (Nejati-Javaremi et al., 2007). Prevention or reduction of tail fat deposition while enhancing lean meat production is the main purpose of the sheep industry. This has been eventually reached by docking at birth the fat tail, slaughtering at an early age, or by crossing the fat-tailed breeds with the thin-tailed breeds (Pourlis, 2011). The diversity of adipocyte volume across breeds is influenced by diet and genetic effects. The genetic effects are considered the main determinants for the formation and structure of adipocytes (Cheng et al., 2016; Davidson, 2014). Therefore, none of the aforementioned methods have been able to significantly reduce the tail fat in fat-tailed sheep.

To date, there have been several studies on the lipid metabolism of tail in different sheep breeds. Miao and Luo (2013) analyzed the transcriptome information of subcutaneous adipose tissue between Small-tailed Han and Dorset sheep. Wang et al. (2014) studied the diversity in the transcriptome profile of tail fat tissue between fat-tailed Kazak sheep and Tibetan short-tailed sheep and identified two top genes (NELL1 and FMO3) with the largest expression differences between the two groups (Wang et al., 2014). Li et al. (2018) investigated the transcriptome of three adipose tissues from Guangling Large-tailed and Small-tailed Han sheep and reported four highly expressed co-genes, FABP4, $A D I P O Q, F A B P 5$, and $C D 36$ which are known as genes with close relation to adipose deposition ( $\mathrm{Li}$ et al., 2018). Moreover, Bakhtiarizadeh et al. (2019) reported several enriched functional terms which might contribute to the deposition of fat in the tail of sheep via comparative transcriptome analysis between fat-tailed (Lori-Bakhtiari) and thin-tailed (Zel) Iranian sheep breeds (Bakhtiarizadeh et al., 2019). As mentioned, there were only a handful of high throughput, comprehensive studies that aimed to decipher the functional genes that differentiate the fat storage pattern of thin- and fattailed sheep breeds. Therefore, the current work was a further attempt to discover the functional genes that are responsible for fat deposition or fat lipolysis in the tail fat of sheep.

Whole transcriptome sequencing is a strong tool for exploring the genetic structure of complex traits (Wickramasinghe et al., 2014). Gene expression patterns may describe phenotypic differences in the tail fat of sheep breeds. Thus, the identification of gene networks and metabolic pathways involved in fat deposition or fat lipolysis could help to improve the quality of sheep meat and carcass (Bernard et al., 2007; Damon et al., 2013).

Knowledge about the molecular mechanism of fat storage is crucial for decreasing the fat mass in the carcass ( $\mathrm{Li}$ et al., 2018). The mechanism of lipid metabolism is complex, and the manipulation of fat storage for lean meat production is very important in the sheep-breeding industry. The present study aimed to study the genetic profiles of fat tissues and to discover the diversity in the genetic mechanisms defining fat deposition between two morphologically different sheep breeds. We characterized the transcriptome of tail fat tissue from Ghezel (fat-tailed) and Zel (thin-tailed) sheep breeds. Analysis of the diversity of fat deposition between these two sheep breeds may aid the recognition of genes and pathways responsible for the formation of tail fat; therefore manipulation of fat deposition can contribute to new breeding strategies. We emphasized the DEGs and pathways involved in lipid metabolism and interpreted how they led to the differences in adiposity between Ghezel (fat-tailed) and Zel (thin-tailed) sheep breeds. 


\section{Materials and methods}

\subsection{Ethics statement}

This study was carried out in strict accordance with the recommendations in the Guide for the Care and Use of Laboratory Animals of the National Institutes of Health. The protocol was approved by the Committee on the Ethics of Animal Experiments of the University of Tabriz, Iran (protocol number: 20170415/39/44).

\subsection{Animals and samples}

Eight healthy male lambs were selected for the experiment; four from the Ghezel (fat-tailed) breed and four from the Zel (thin-tailed) breed. All lambs were weaned at $90 \mathrm{~d}$ of age and fostered under similar conditions at the research station of the Faculty of Agriculture, University of Tabriz, Tabriz, Iran (Khalatpoushan). The lambs were housed in individual pens and provided the same diet ad libitum for $120 \mathrm{~d}$ and were slaughtered at age 7 months. The adipose tissues in the tail were rapidly collected under sterile conditions, instantly frozen in liquid nitrogen, and stored at $-80^{\circ} \mathrm{C}$ until total RNA isolation.

\subsection{RNA extraction}

Total RNA was isolated from the tail adipose tissues using a Trizol reagent according to the manufacturer's instructions (TaKaRa, USA). In essence, fat tissues were powdered using liquid nitrogen, homogenized and centrifuged at $12000 \mathrm{~g}$ for $10 \mathrm{~min}$ at $4{ }^{\circ} \mathrm{C}$ to remove the insoluble material. Following the elimination of the top fatty layer on the aqueous phase, a clear supernatant was used for the next RNA extraction step. NanoDrop (Thermo Scientific NanoDrop 2000) was used to check the quantity of total RNA, and the $28 \mathrm{~S} / 18 \mathrm{~S}$ ratio was evaluated by electrophoresis on $1 \%$ agarose gel to detect the RNA integrity. Finally, RNA samples that had a $28 \mathrm{~s} / 18 \mathrm{~s}$ ratio greater than 1 and an OD $260 \mathrm{~nm} / \mathrm{OD} 280 \mathrm{~nm}$ ratio more than 1.9 were selected for sequencing. The integrity and concentration of RNA were measured using the 2100 Bioanalyzer (Agilent Technologies, Waldronn, Germany). The RNA integrity number value of all samples was above 7.0.

\subsection{RNA sequencing and library preparation}

Library preparation started with DNase I treatment, then poly(A) enrichment using oligo (dT) magnetic beads (Invitrogen, USA). The fragmentation step was conducted with short mRNA fragments which were subsequently used as templates for the first-strand cDNA synthesis. For the second-strand cDNA production, short fragments were ligated to adaptors with added poly(A) tails. After agarose gel electrophoresis, suitable fragments for PCR amplification were selected as templates. Unfortunately, one of the Ghezel (fat-tailed) samples failed in the library preparation step.
Therefore, only seven paired-end cDNA libraries (four from Zel (thin-tailed) and three from Ghezel (fat-tailed) breeds) were produced. Finally, the sequencing of the libraries for the generation of $150 \mathrm{~b}$ paired-end reads was carried out using the Illumina HiSeq2000 platform. The generated raw RNASeq data were deposited into a NCBI SRA database with BioProject accession number PRJNA598581.

\subsection{Quality control, mapping and quantification}

FastQC (v0.11.5) was used for quality control of raw sequencing reads (Andrews, 2010). Trimmomatic software (v0.35) was used to trim the raw reads with adapter contamination, as well as for reads with more than $10 \%$ of unknown bases and for reads with more than $50 \%$ of lowquality bases (quality value $\leq 10$ ) (Bolger et al., 2014). Finally, reads longer than 90 bp with a minimum Phred score of 20 were kept for the analyses. The clean reads were mapped to the sheep reference genome $\mathrm{v} 4.0$ (ftp://ftp.ncbi. nlm.nih.gov/genomes/Ovis_aries, last access: June 2020) using Bowtie (v2.2.4) (Langmead and Salzberg, 2012), SAMtools (v1.3.1) (Li et al., 2009) and TopHat (v2.1.1) (Trapnell et al., 2009). Assembled reads by Cufflinks (v2.2.1) were annotated with the NCBI reference annotation (ftp://ftp.ncbi. nlm.nih.gov/genomes/Ovis_aries) (Trapnell et al., 2012). Using Cuffmerge, individual transcripts were merged to form a single transcript assembly, and using Cuffdiff (v2.2.1), the merged transcript was used for quantification and differential expression analysis.

\subsection{Gene expression analysis}

Fragments per kilobase of exon per million fragments mapped (FPKM) values were used for the gene expression analysis. The FPKM for gene abundances was normalized for the library and gene length by comparing to an annotated reference genome (Oar_v4.0) (Mortazavi et al., 2008).

Gene expression analysis in the Ghezel (fat-tailed) and Zel (thin-tailed) breeds was performed with Cuffdiff. All differentially expressed genes with a $\log 2$ fold change larger than 1.1 at $q$ value $<0.05$ were selected. To obtain the similarity profile of gene expression in biological replicates of two breeds, principal component analysis (PCA) was conducted using web-based ClustVis tools (https://biit.cs.ut.ee/clustvis/, last access: July 2020).

\subsection{GO classification analysis and KEGG pathway}

We carried out the functional enrichment analysis for upand down-regulated DEGs, separately. Therefore, in order to identify three categories of gene ontology (GO) including the biological processes, cellular components and molecular functions terms, the DEGs were submitted to the Enrichr Database (https://amp.pharm.mssm.edu/Enrichr/, last access: August 2020). Kyoto Encyclopedia of Genes and Genomes 
pathway analysis (http://www.genome.jp/kegg/, last access: August 2020) was used for pathway analysis. The adjusted $p$ value $<0.05$ was considered as the cutoff threshold for GO and KEGG pathway analyses.

\subsection{Protein-protein interaction network and module analysis}

To gain a further comprehension of the biological relationships between genes, the DEG list was inputted into the STRING database (https://string-db.org/, last access: September 2020). To define the functional modules, the constructed networks were clustered with the $K$ mean algorithm to the three modules. Also, Cytoscape plug-in cytoHubba (v 3.7.2) was applied to detect the hub genes with the maximal clique centrality (MCC) method.

\subsection{Structural classification of proteins}

To find the structural class of genes related to lipid storage, total DEGs were imported to pathway studio web mammal version 11.2 (https://www.pathwaystudio.com/, last access: August 2020).

\subsection{Validation of data}

To confirm the different genes expressed, we retrieved the data of a similar study from the Gene Expression Omnibus (GEO) (https://www.ncbi.nlm.nih.gov/geo/, last access: October 2020) database, and gene expression analyses were conducted as above. Finally, the results were compared with our results to confirm the results of the current experiment.

\section{Results}

\subsection{Sequencing data and mapping summary}

A total of $\sim 47$ gigabases containing 318081210 pairedend raw reads were generated. We obtained approximately 45 million paired-end clean reads of $150 \mathrm{bp}$ for each sample and high percentages of mapped reads ranging from $82.40 \%$ to $87.10 \%$. A mean of $85.17 \%$ of clean reads was mapped to the sheep genome sequence (Ovis aries $\mathrm{v} 4.0$ ). Clean reads included $6.5 \%-10.4 \%$ non-uniquely mapped reads and $12.9 \%-17.6 \%$ unmapped reads. The summary of the mapping of samples is presented in Table 1.

At first, PCA analysis of gene expression in the Zel (thintailed) and Ghezel (fat-tailed) breeds was performed to show the variance of the biological replicates of each breed. PCA results showed the distinct difference between the transcriptome profiles of Zel (thin-tailed) and Ghezel (fat-tailed) breeds (Fig. 1). PC analysis was also performed for the structural protein class of DEGs, and the transporter class showed better diversity between two breeds.

According to the expression values, genes were classified into three groups including low expression ( $\leq 10$ FPKM), moderate expression $(10<\mathrm{FPKM} \leq 500)$ and high expression (> 500 FPKM). Transcriptome results showed that, in both breeds, most of the genes belonged to the low expression class (Table 2).

\subsection{Analysis of some highly expressed genes}

Mapped reads abundances were normalized for library size and gene length (FPKM) to determine unbiased gene expression. Figure 2 shows the top 10 highly expressed genes (>500 FPKM) for the Ghezel (fat-tailed) and Zel (thintailed) breeds. For both breeds, the top 10 highly expressed genes contributed to approximately $87 \%$ of the total reads, which means that a small number of genes possessed a big portion of the total RNA of adipose tissue.

\subsection{Expression level of differentially expressed genes in Ghezel (fat-tailed) and Zel (thin-tailed) breeds}

A total of 332 genes were found to be differentially expressed between Ghezel (fat-tailed) and Zel (thin-tailed). Out of the 332 DEGs, 78 were up-regulated, whereas the remaining 254 were down-regulated in the Zel (thin-tailed) breed. Total DEGs are presented in an additional spreadsheet file (see Table S1 in the Supplement). A volcano plot of the expressed genes is shown in Fig. 3. Also, the heatmap of the top 50 DEGs is shown in Fig. 4. A heatmap is a simple guide to assess the characteristics of RNA-seq results in which the various colors represent the level of differential expression. The red color represents a higher expression, and the blue color represents the lower expression.

The top 10 up- and down-regulated DEGs were sorted in Table 3.

\subsection{Functional enrichment analysis}

Gene ontology analysis of DEGs was done for cellular components, molecular function, and biological process categories using the web-based Enrichr tool. The results were reported as significant in the case that adjusted $p<0.05$. The total terms that were enriched in the cellular components and molecular function category for total DEGs and biological processes for up- and down-regulated DEGs are presented in four supplemental spreadsheet files (see Tables S2, S3, S4 and S5, respectively).

The cell component terms of "extracellular exosome", "mitochondrion", "peroxisome" and "extracellular matrix" as well as molecular function terms of "NAD binding", "oligopeptide transporter activity" and "RNA polymerase II core promoter proximal region sequence-specific DNA binding" were significantly enriched with DEGs. Up- and downregulated DEGs were mapped separately onto the KEGG pathway database to identify the related biological pathways with fat deposition (Fig. 5). For the 254 down-regulated DEGs in the Zel (thin-tailed) breed, $127 \mathrm{GO}$ terms were 
Table 1. Summary of RNA-seq data and mapping statistics of adipose samples of Zel (thin-tailed) and Ghezel (fat-tailed) sheep breeds.

\begin{tabular}{|c|c|c|c|c|c|c|c|}
\hline Samples & Zel-1 & Zel-2 & Zel-3 & Zel-4 & Ghezel-1 & Ghezel-2 & Ghezel-3 \\
\hline Total & 4791905 & 439099 & 4288 & 4333 & 437 & 45 & 510 \\
\hline Total b & 187858100 & 586489800 & 432507600 & 499589700 & 6565108800 & 678364 & 7656982800 \\
\hline Total mapped reads & $\begin{array}{r}37339712 \\
(84.3 \%)\end{array}$ & $\begin{array}{r}35103330 \\
(87.1 \%)\end{array}$ & $\begin{array}{r}33763526 \\
(85.5 \%)\end{array}$ & $\begin{array}{r}34616084 \\
(86.7 \%)\end{array}$ & $\begin{array}{r}35112150 \\
(86.8 \%)\end{array}$ & $\begin{array}{r}34757959 \\
(82.4 \%)\end{array}$ & $\begin{array}{r}39097097 \\
(83.4 \%)\end{array}$ \\
\hline quely mapped reads & 5389716 & 2323331 & 2212652 & 2297504 & 2371892 & 2880142 & 4049800 \\
\hline Unmapped reads & 10579342 & 8806602 & 9119858 & 8714514 & 8655242 & 10466339 & 11949455 \\
\hline
\end{tabular}
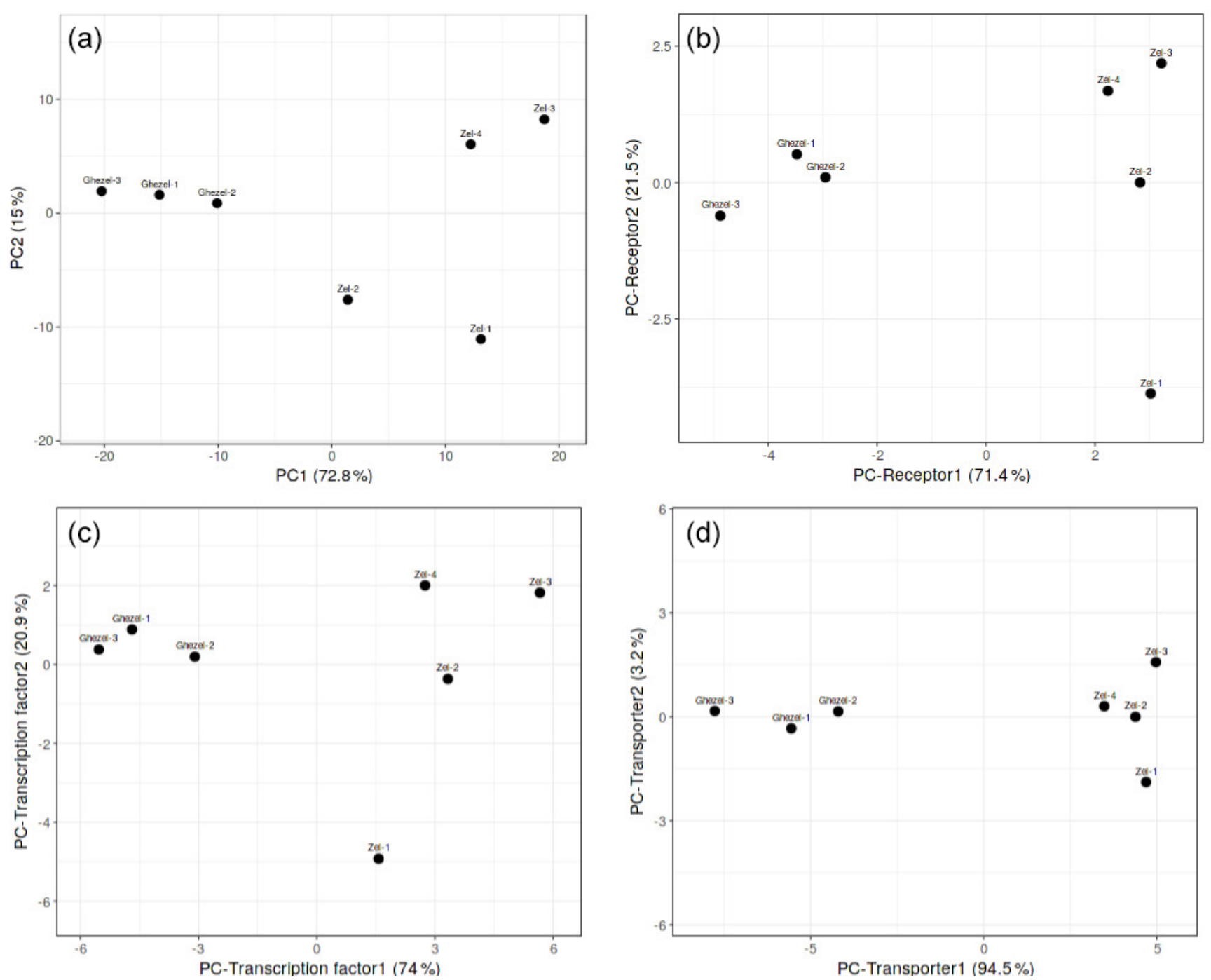

Figure 1. PCA scatter plot of differentially expressed genes in adipose tissues between Zel (thin-tailed) and Ghezel (fat-tailed) sheep breeds: (a) total differentially expressed genes, (b) differentially expressed receptors, (c) differentially expressed transcription factors, (d) differentially expressed transporters.

significant (adjusted $p<0.05$ ), with most of the terms associated with fat metabolism. These are "monocarboxylic acid metabolic process", "dicarboxylic acid metabolic process", "acyl-CoA metabolic process", "fatty acid metabolic process", "cholesterol biosynthetic process", "tricarboxylic acid metabolic process", "glycerophospholipid biosynthetic process", "membrane lipid metabolic process", "fatty acid biosynthetic process", "fatty-acyl-CoA metabolic process", "fatty acid derivative biosynthetic process" and "longchain fatty-acyl-CoA metabolic process". The KEGG pathway analysis of down-regulated genes revealed 27 significant pathways (adjusted $p<0.05$ ) that had similar pat- 
Table 2. Distribution of genes of different samples of Zel (thin-tailed) and Ghezel (fat-tailed) sheep breeds in three expression categories (high, moderate and low).

\begin{tabular}{lrrrr|rrr}
\hline Samples & \multicolumn{3}{c|}{ Zel (thin-tailed) } & \multicolumn{3}{c}{ Ghezel (fat-tailed) } \\
\cline { 2 - 8 } & Zel-1 & Zel-2 & Zel-3 & Zel-4 & Ghezel-1 & Ghezel-2 & Ghezel-3 \\
\hline High expressed genes $(>$ 500 FPKM) & 503 & 450 & 690 & 635 & 493 & 508 & 488 \\
Moderate expressed genes $(10<$ FPKM $\leq 500)$ & 6398 & 7196 & 9923 & 8436 & 7214 & 6637 & 6088 \\
Low expressed genes $(\leq 10$ FPKM) & 16164 & 18121 & 17459 & 18652 & 18900 & 15801 & 15862 \\
Total expressed genes & 23065 & 25767 & 28072 & 27723 & 26607 & 22946 & 22438 \\
Total unexpressed genes & 11287 & 11239 & 14767 & 13674 & 11800 & 11084 & 11224 \\
\hline
\end{tabular}
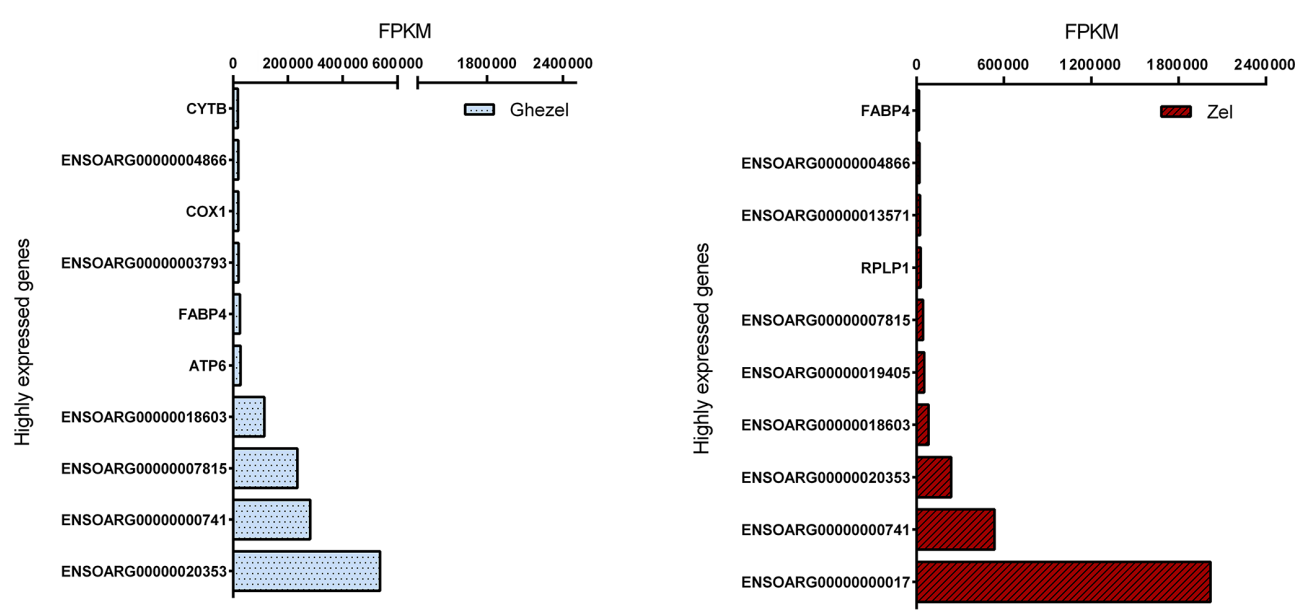

Figure 2. Top 10 highly expressed genes in adipose tissue of Ghezel (fat-tailed) and Zel (thin-tailed) breeds based on FPKM value.

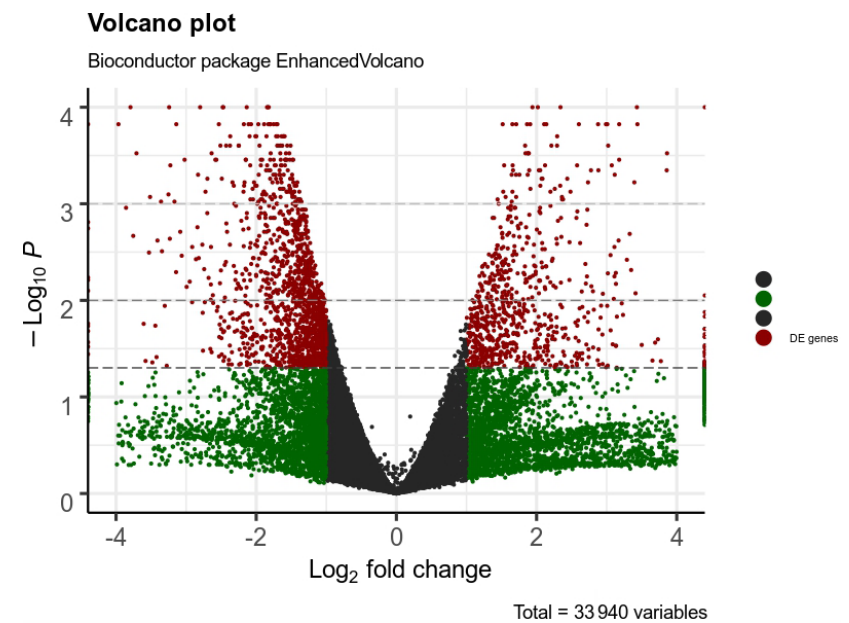

Figure 3. Volcano plot of expressed genes of adipose tissue of Zel (thin-tailed) compared to Ghezel (fat-tailed) sheep breeds. The $y$ axis illustrates $-\log _{10} p$ values, and the $x$ axis corresponds to a $\log _{2}$-fold change of gene expression between Zel and Ghezel sheep breeds. The red points represent significant genes ( $p$ value $<0.05$ ). terns to GO terms. These include "fatty acid biosynthesis", "fatty acid metabolism", "steroid biosynthesis", "propionate metabolism", and "pyruvate metabolism". Furthermore, enrichment analysis of 78 up-regulated DEGs in the Zel (thintailed) breed showed 191 significant biological processes and $30 \mathrm{KEGG}$ pathways that are related to fat metabolism. Some GO biological process terms for these genes such as "positive regulation of stress-activated mitogen-activated protein kinase (MAPK) cascade", "activation of MAPKKK activity" and "positive regulation of p38MAPK cascade" are related to the "MAPK signaling pathway" which is activated during adipocyte lipolysis with rapid stimulation of hormonesensitive lipase (HSL) activity.

\subsection{Protein-protein interaction (PPI) network and module analysis}

To reach a greater understanding of the biological relationships between the genes of a complex process such as fat deposition, the information about the functions of genes and proteins is required. Therefore, protein-protein interaction analysis is necessary. The up- and down-regulated DEGs were analyzed separately with the STRING database (v 10.5) (https://string-db.org/) (Szklarczyk et al., 2016). Of 332 DEGs, 300 were annotated in the STRING database 


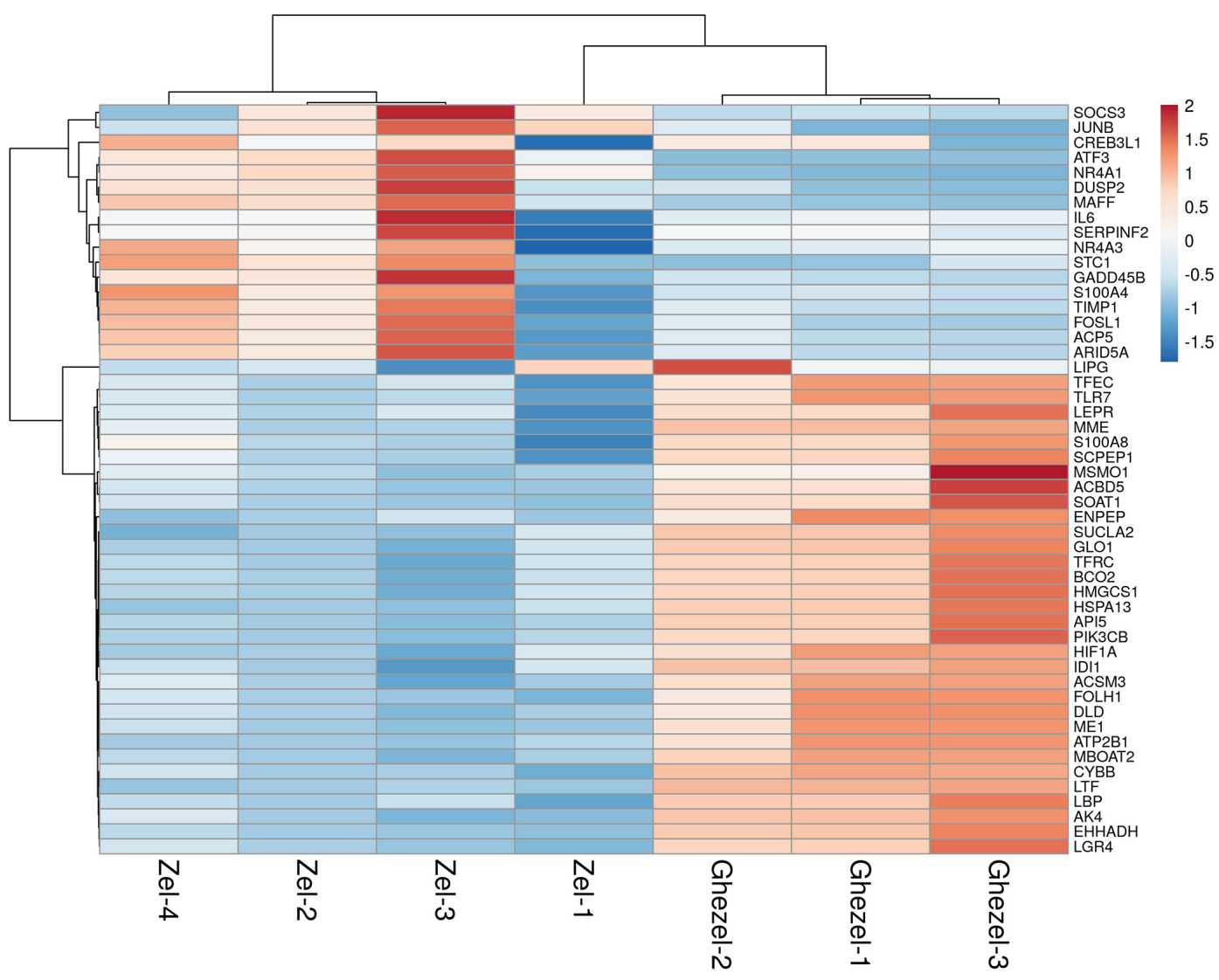

Figure 4. Expression profile of the 50 top differentially expressed genes in adipose tissue between Zel (thin-tailed) compared to Ghezel (fat-tailed) sheep breeds. Up- and down-regulated genes are separately colored with red and blue.

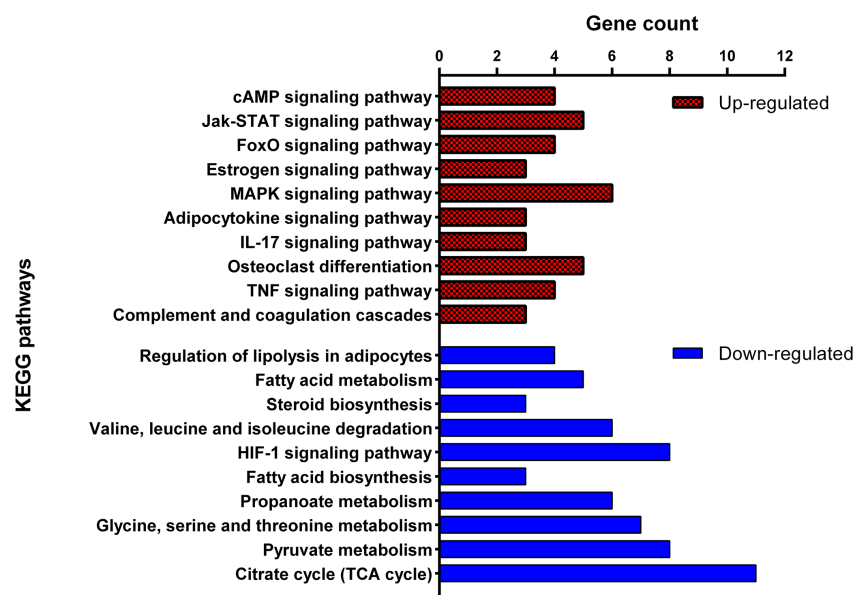

Figure 5. Top 10 significant Kyoto Encyclopedia of Genes and Genomes (KEGG) pathways enriched by up-regulated (red) and down-regulated (blue) differentially expressed genes of adipose tissue of Zel (thin-tailed) compared to Ghezel (fat-tailed) sheep breeds. and utilized to construct the PPI network. Enrichment analysis showed that PPI networks were significantly enriched (adjusted- $p<0.05$ ). Text mining, databases, gene fusion, co-expression, and neighborhood interactions were considered for the network construction. Disconnected nodes were deleted in the PPIs and scores of interaction confidence (confidence score $<0.4$ ) were considered. To define the functional modules, the constructed networks were clustered with the $K$ mean algorithm to the three modules. $K$ mean is a common clustering algorithm that classifies a dataset into $K$ (a predefined number) categories (Le and Kim, 2015). The Cytoscape plug-in cytoHubba (v 3.7.2) was applied to detect the hub genes with the MCC method. MCC has better accuracy for predicting principal proteins and hub genes in the PPI network (Chin et al., 2014; Chen et al., 2009). Hub genes have a main effect on the network topology (Albert and Barabási, 2002).

In Fig. 6, the up-regulated DEGs in the red module (PPI enrichment $p$ value: $3.92 \times 10^{-11}$ ) included significant functional terms such as osteoclast differentiation (false discovery rate, $\mathrm{FDR}=0.00060$ ), insulin resistance $(\mathrm{FDR}=0.0123), \mathrm{TNF}$ signaling pathway $(\mathrm{FDR}=0.0123)$, $I L-17$ signaling pathway $(\mathrm{FDR}=0.0123)$, complement and 
Table 3. Top 10 up- and down-regulated genes in adipose tissue of Zel (thin-tailed) compared to Ghezel (fat-tailed) sheep breeds.

\begin{tabular}{llr}
\hline Ensemble gene ID & Gene name & $\begin{array}{r}\log _{2} \\
\text { (fold change) }\end{array}$ \\
\hline Up-regulated genes & & \\
\hline ENSOARP00000014659 & IL6 & 6.98 \\
ENSOARG00000017834 & Unnamed & 6.30 \\
ENSOARG00000004007 & LIPG & 5.66 \\
ENSOARG00000018812 & HSPB7 & 5.42 \\
ENSOARG00000019972 & SCG5 & 3.86 \\
ENSOARG00000009963 & SAA1 & 3.62 \\
ENSOARG00000010042 & STC1 & 3.56 \\
ENSOARG00000009119 & CCL19 & 3.44 \\
ENSOARG00000014064 & NR4A3 & 3.39 \\
ENSOARG00000000175 & SOCS3 & 3.36 \\
\hline Down-regulated genes & & \\
\hline ENSOARG00000014064 & Unnamed & 13.52 \\
ENSOARG00000020086 & SLC15A2 & 6.09 \\
ENSOARG00000008620 & LTF & 5.71 \\
ENSOARG00000017865 & DNHD1 & 5.10 \\
ENSOARG00000020354 & MOGAT1 & 4.89 \\
ENSOARG00000018816 & LBP & 4.69 \\
ENSOARG00000001217 & CYP4F21 & 4.47 \\
ENSOARG00000014789 & MBOAT2 & 4.28 \\
ENSOARG00000010196 & TNFRSF9 & 3.97 \\
ENSOARG00000003714 & ENPEP & 3.78 \\
\hline
\end{tabular}

coagulation cascades $(\mathrm{FDR}=0.0123)$, Jak-STAT signaling pathway $(\mathrm{FDR}=0.0125)$, and cytokine-cytokine receptor interaction $(\mathrm{FDR}=0.0200)$. DEGs in the blue module (PPI enrichment $p$ value: $8.45 \times 10^{-9}$ ) were included p53 signaling pathway $(\mathrm{FDR}=0.00074)$, FoxO signaling pathway $(\mathrm{FDR}=0.00074)$ and MAPK signaling pathway $(\mathrm{FDR}=0.0016) . I L-6$ and $J U N B$ genes were also found as hub genes in the red and green module of up-regulated PPI, respectively.

In Fig. 7, the down-regulated DEGs in the green module (PPI enrichment $p$ value: 0.0127 ) were significantly enriched in functional terms such as fatty acid biosynthesis $(\mathrm{FDR}=0.00022)$, fatty acid metabolism $(\mathrm{FDR}=0.00044)$, propionate metabolism $(\mathrm{FDR}=0.00057)$ and pyruvate metabolism $(F D R=0.00057)$. Significant terms in the green module were more strongly associated with fat metabolism than the other modules. DEGs in the red module (PPI enrichment $p$ value: $5.55 \times 10^{-16}$ ) were related to glycine, serine and threonine metabolism $(\mathrm{FDR}=9.58 \times$ $\left.10^{-5}\right)$; pyruvate metabolism $(\mathrm{FDR}=0.00049)$; glycolysis/gluconeogenesis $(\mathrm{FDR}=0.0030)$; and HIF-1 signaling pathways $(\mathrm{FDR}=0.0167)$, as well as biosynthesis of amino acids (FDR $=0.0246$ ), biotin metabolism $(\mathrm{FDR}=0.0257)$ and regulation of lipolysis in adipocytes $(F D R=0.0472)$. DEGs in the blue module (PPI enrichment $p$ value: $1.0 \times 10^{-16}$ ) were related to steroid biosynthesis $\left(\mathrm{FDR}=1.25 \times 10^{-11}\right)$ and terpenoid backbone biosynthesis (FDR $=0.00021)$. Also, the HMGCS1 gene was identified as a hub gene for the green module, VPS35 as a hub gene for the blue module and VPS26A as a hub gene for the red module in down-regulated PPI.

\subsection{Structural classification of proteins}

Total DEGs were imported to a web-based pathway studio. Out of 332 DEGs, 107 were present in seven classes, including transporter, transcription factor, receptor, ligand, protein kinase, protein phosphatase and pseudogene. Expression patterns of the DEGs were mainly classified as genes encoding transporters, transcription factors and receptors, respectively. PCA results demonstrated that most of the DEGs in each of the aforementioned three clusters showed a distinct expression profile of tail fat in two sheep breeds (Fig. 1). Also, some of the annotated DEGs in the transcription factor class, such as JUNB, NR4A3, HIF-1 $\alpha, F O S L 1, M A F F$, $N R 4 A 1, C R E B 3 L 1$, and ATF3 were related to lipolysis function. More interesting is that all aforementioned genes except the $H I F-l \alpha$ gene were up-regulated in the Zel (thin-tailed) breed. Gene expression is regulated by activating or suppressing transcription factors, so the aforementioned DEGs may have stimulated lipolysis of fat in the tail of Zel (thintailed) sheep.

\subsection{Validation of differentially expressed genes}

To validate the DEGs of the current study we reanalyzed the data of the Bakhtiarizadeh et al. (2019) study. The Venn diagram of the DEGs showed 35 common DEGs between the two studies (Fig. 8). Of the 35 common genes between the two studies, 28 genes showed the same direction expression and 7 genes demonstrated opposite direction of expression. Their common genes and expression direction are presented in a supplemental spreadsheet file (see Table S6). Among the DEGs that were validated by q-RT-PCR in the study of Bakhtiarizadeh et al. (2019), we detected three common DEGs including S100A8, TNNCl and JUNB genes. Two out of those three genes showed the same expression patterns (Fig. 9).

\section{Discussion}

The mechanism of fat deposition is complex and the improvement of meat production is of great importance in sheep breeding. The main goal of the present study was to define the genetic mechanisms underlying lipid storage in the tail of Iranian sheep breeds. The comparison of two morphologically different sheep breeds was made with regard to the effect of breed on fat deposition. In our study, samples of tail fat tissues were collected at the end of adipocyte differentiation stage. So, the higher expression level in the 


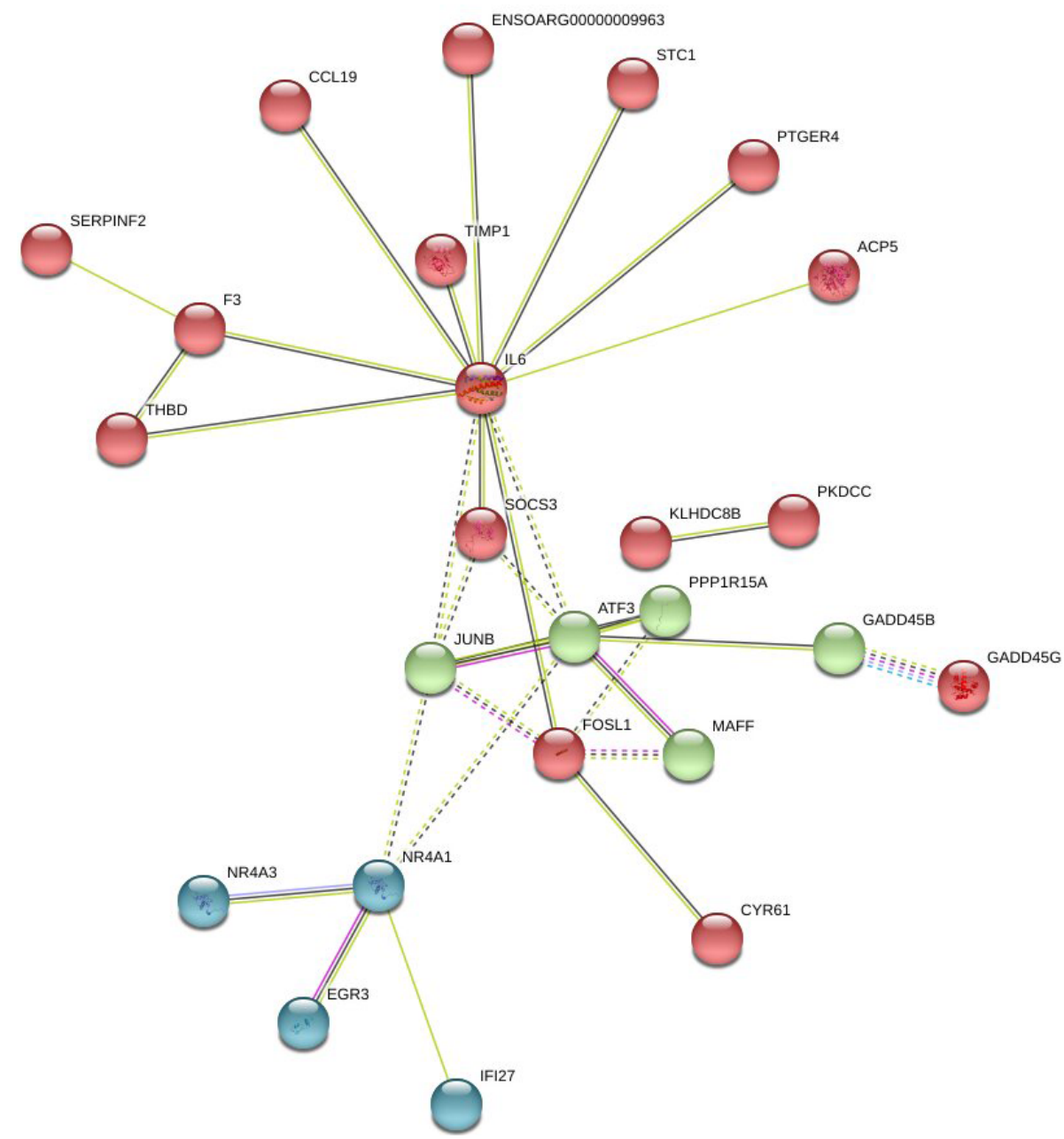

Figure 6. Protein-protein interaction (PPI) network and functional module analysis of up-regulated differentially expressed genes of adipose tissues of Zel (thin-tailed) compared to Ghezel (fat-tailed) sheep breeds.

adipocytes of the fat-tailed breed demonstrates the stronger role of these genes in adipose metabolism than in thin-tailed sheep breeds. Classification of proteins also showed that, out of 35 DEGs in the transporter class, 11 DEGs belonged to the SLC families. SLC transporters are the largest transporter family and responsible for transporting inorganic ions, amino acids and lipids across the membrane. All these genes were up-regulated in the Ghezel (fat-tailed) breed. Therefore, it can be postulated that the overexpression of SLC family genes in the Ghezel (fat-tailed) breed might contribute to the accumulation of fat in the tail.

Out of the top 10 highly expressed genes, 4 were in common in both breeds, including ENSOARG00000000741, ENSOARG00000007849, ENSOARG00000020353 and ENSOARG00000018603. It seems that these genes have an important, determinant role in the metabolism of adipose tissue. Furthermore, fatty acid binding protein 4 $(F A B P 4=$ ENSOARG00000009344 $)$ and cytochrome c oxidase $1(C O X 1=$ ENSOARG00000000016) genes may play an important role in the regulation of fat deposition in the Ghezel (fat-tailed) breed. FABP4 is a well-known gene that is involved in lipid metabolism, and it has been demonstrated that its plasma level is progressively increased according to body mass index (BMI) (Queipo-Ortuño et al., 2012) and shows a positive correlation with body weight $(r=0.377)$ (Auguet et al., 2014). Furthermore, recent studies have shown that $C O X$ pathways may be involved in both increased size and the number of mature adipocytes in the differentiation pre-adipocyte process. These results suggested that two isoforms of $C O X$ had different functions: with $C O X 2$, which is involved in body fat regulation, and COX1, whose role is not yet determined (Yan et al., 2003). As a result, although further research is needed to determine the effect of COX1 and FABP4 genes on the accumulation of fat in the tail, the higher expression of both COX1 and FABP4 genes in the Ghezel (fat-tailed) than in the Zel (thin-tailed) breed might suggest a potential role for them in 


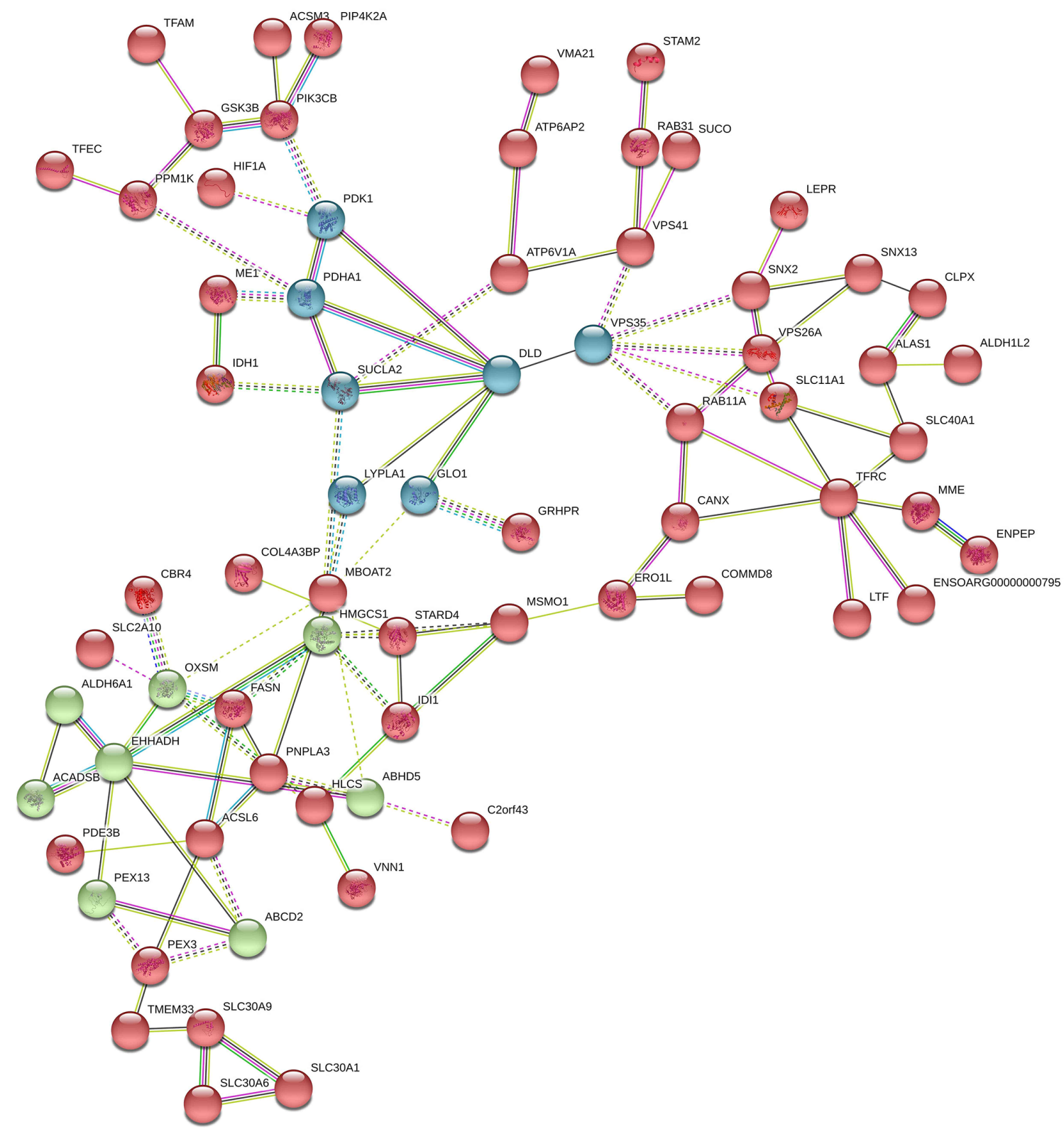

Figure 7. Protein-protein interaction (PPI) network and functional module analysis of down-regulated differentially expressed genes of adipose tissues of Zel (thin-tailed) compared to Ghezel (fat-tailed) sheep breeds.

the deposition of excessive fat in the tail of fat-tailed sheep breeds (Fig. 2).

Results of up- and down-regulated DEGs show that some down-regulated DEGs in the Zel (thin-tailed) breed such as $L T F, L B P, M O G A T 1$ and MBOAT2 are related to lipid storage. Min et al. (2018) reported that lactoferrin (LTF) has favorable effects on body weight and lipid accumulation in mice. In another study the up-regulation of LTF has been observed in Small-tailed Han sheep as compared with that of Tibetan thin-tailed sheep (Ma et al., 2018). Up-regulation of LTF in the Ghezel (fat-tailed) breed suggests that this gene has a key function in fat deposition in fat-tailed sheep breeds.
Another down-regulated DEG in the Zel (thin-tailed) breed is lipid-binding protein-5 (LBP-5) gene. As a result, the lipidbinding protein family is essential for homeostasis of fatty acid and lipid transfer (Moreno-Navarrete et al., 2017), and $L B P-5$ regulates fat accumulation in Caenorhabditis elegans and is bound directly to fatty acids with varying affinities (Xu et al., 2011). Indeed, FABPs are members of the intracellular LBP that have a role in the accumulation of fat (Zheng et al., 2013). Similar to LBP-5, the expression of the monoacylglycerol $O$-acyltransferase 1 (MOGATI) gene is inversely correlated with the lipolytic rate, and its suppression results in the enhancement of basal lipolytic activity (Liss et al., 


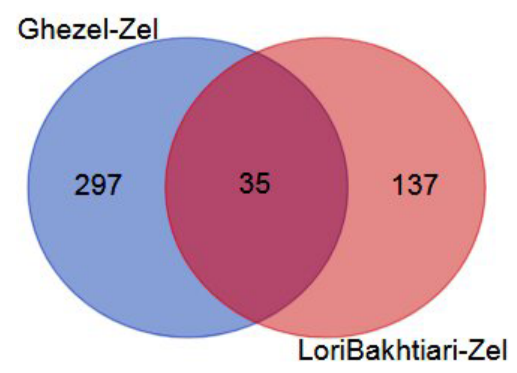

Figure 8. Venn diagram of the differentially expressed genes in the current study (Ghezel vs. Zel comparison) and Bakhtiarizadeh et al. (2019) study (Lori-Bakhtiari vs. Zel comparison).

2018; Shi et al., 2019). So this result suggests that the upregulation of the aforementioned genes can make more fat deposition in the tail of the Ghezel (fat-tailed) breed (Table 3). Some up-regulated DEGs in the Zel (thin-tailed) breed are associated with fat lipolysis, such as IL6, SAAI, LIPG, NR4A3 and SOCS3 (Table 3). As a result, both inhibitions of lipolysis and the promotion of lipogenesis could lead to the increment of fat in the tail of Ghezel (fat-tailed) sheep. In contrast, the overexpression of the aforementioned genes in the thin-tailed breeds may result in a smaller tail. One of the important genes in the lipolysis of fatty acids is nuclear receptor subfamily 4 group A member 3 (NR4A3). There have been reports that the overexpression of NR4A3 produced a marked reduction in body weight in mice and several insulin-resistant rodent models (Walton et al., 2016). Similar to $N R 4 A 3$, endothelial lipase ( $L I P G$ ) is a form of lipase that is secreted by vascular endothelial cells and plays an important role in lipoprotein metabolism and the lipid composition of cells (Justine et al., 2018). Lipase characteristics of this gene suggest a significant pivotal degradation role for decreasing fat deposition. Another up-regulated gene in the Zel (thin-tailed) breed was serum amyloid A1 (SAAl), which has been reported to play a critical role in the $\beta$ oxidation of lipids as well as in promoting lipolysis and inhibiting the expression of genes related to lipogenesis. SAAl inhibited the expression of perilipin which acts as a protective coating from the body's natural lipases, such as hormone-sensitive lipase (HSL) (Wang et al., 2010). Like other up-regulated DEGs, interleukin-6 (IL-6) participates in the metabolism of fat, which elevates lipolysis and the release of liver free fatty acids and triglycerides (Zhang et al., 2014). IL-6 affects lipid metabolism and, being a lipolytic factor, stimulates the lipolysis and oxidation of fatty acids in humans (van Hall et al., 2003; Xu et al., 2018), bovines (Contreras et al., 2017b) (Contreras et al., 2017a), mice (Han et al., 2018; Ma et al., 2015) and rats (Nonogaki et al., 1995). But the role of $I L-6$ in lipolysis of the fat in sheep has not yet been fully elucidated. Up-regulation of this gene may contribute to the lipolysis of fatty acids in the tail of the Zel (thin-tailed) breed. $I L-6$ is, also, a hub gene in the red module of the PPI network of up- regulated genes (Fig. 6) with a pivotal interaction with other DEGs. These findings suggest an important role for aforementioned genes in decreasing fat deposition in the tail of the Zel (thin-tailed) breed. $I L-6$ was also enriched in several significant GO terms including "positive regulation of cellular process" and "regulation of signaling receptor activity".

Results of enrichment analysis revealed that several pathways are involved in lipolysis such as tumor necrosis factor (TNF) signaling and MAPK signaling pathways. $I L-6$ gene is one of the important genes that enriched the mentioned pathways. MAPK is the main signal-regulating lipid metabolism. Activation of MAPK inhibits fat synthesis and promotes fatty acid oxidation (Grisouard et al., 2012). Interestingly, the IL-6 gene has a strong role in MAPK signaling and TNF signaling pathways, because $I L-6$ promotes lipid breakdown, glycolysis and fatty acid oxidation in skeletal muscle and adipose tissues via the MAPK signaling pathway. Ruderman et al. (2006) showed that $I L-6$ is mainly found in adipose tissue and the center of the hypothalamus and regulates the body fat composition. The deletion of the $I L-6$ gene in mice led to obesity and insulin resistance (Matthews et al., 2010; Duszka et al., 2013). In general, most of the pathways related to lipolysis have $I L-6$ in common. Other enriched DEGs in the MAPK pathway such as nuclear receptor subfamily 4 group A member 1 (NR4Al) (Zhao et al., 2018), dual specificity phosphatase 1 (DUSP1) (Wu et al., 2015), growth arrest and DNA damage-inducible beta $(G A D D 45 B)$ (Kim et al., 2014), and growth arrest and DNA damage-inducible gamma $(G A D D 45 G)$ (Fuhrmeister et al., 2016) were associated with lipid metabolism. Up-regulation of these genes may result in an increased lipolysis in Zel (thin-tailed) sheep. One of the other KEGG signaling pathways that is enriched by IL-6 is the TNF signaling pathway, which removes lipid droplets of perilipin and activates hormone-sensitive lipase (HSL) and adipose triglyceride lipase (ATGL). Both HSL and ATGL release free fatty acids by acting on triacylglycerol and diacylglycerol (Yang et al., 2011). Other up-regulated DEGs that are enriched in the TNF signaling pathway are CAMP responsive element binding protein 3 like 1 (CEREBL1), JunB proto-oncogene, AP-1 transcription factor subunit (JUNB) and suppressor of cytokine signaling (SOCS3), related to lipolysis of fat. The TNF signaling pathway is well-known to increase adipocyte lipolysis (Feingold et al., 1992; Green et al., 1994; Hauner et al., 1995; Kawakami et al., 1987). A recent study demonstrated that SOCS3 plays a critical role in the regulation of fatty acid $\beta$ oxidation and is an important factor for lipid metabolism (Luo et al., 2011). Interestingly, our findings showed a strong interaction between $I L$ 6 and SOCS3 genes. The CREB3L1 gene has also been reported to increase glycerol release and has significant effects on lipolysis by participating in the CAMP signaling pathway. According to Ehrlund et al. (2017), CREB3L1 knockdown led to reduced glycerol release and had significant effects on lipolysis. Wang et al. (2016) investigated the involvement of CREB3L1 in the maintenance of lipid homeostasis and 

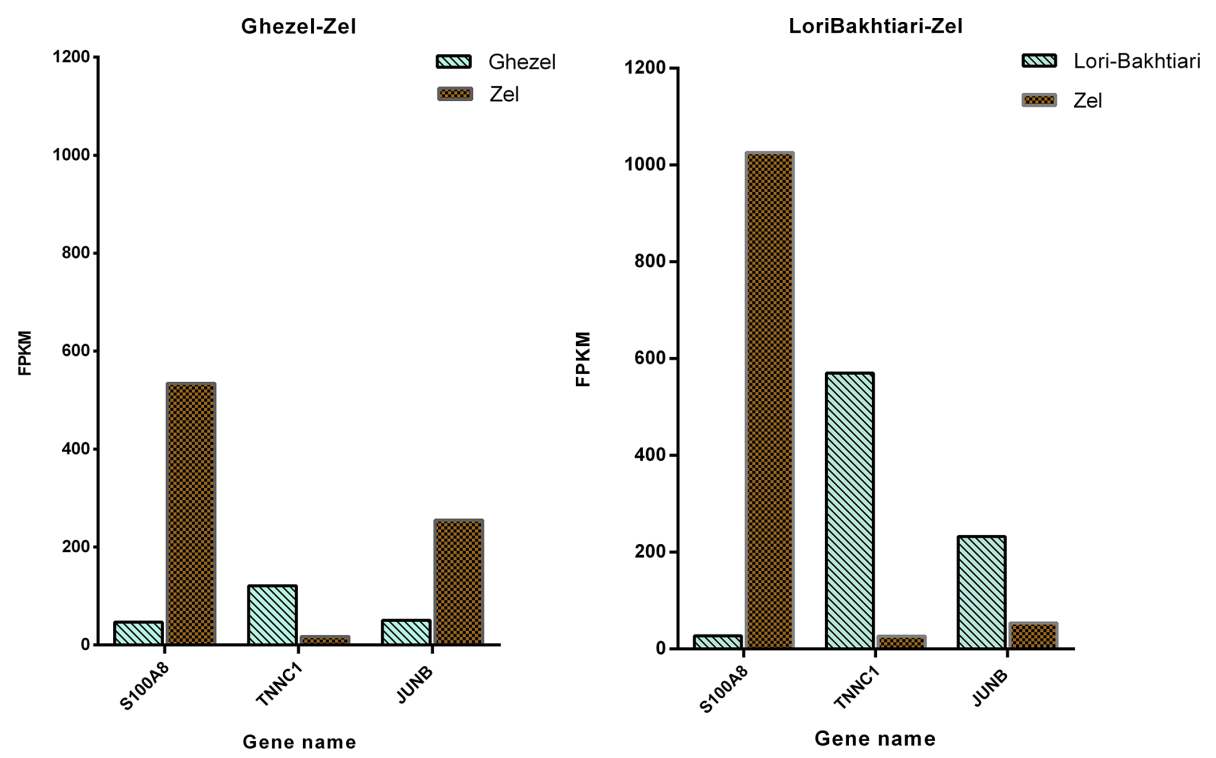

Figure 9. Gene expression levels of three differentially expressed genes in the current study and Bakhtiarizadeh et al. (2019) study. $y$ axis illustrates the expression levels of three genes based on FPKM value.

found that CREB3L1 deletion leads to a defect in triglyceride (TG) lipolysis, resulting in higher levels of plasma TG. Up-regulation of these genes may enhance adipocyte lipolysis and increase the concentrations of circulating free fatty acids in the Zel (thin-tailed) breed. The results of the current work may suggest a crucial role for DEGs related to TNF signaling and MAPK signaling pathways, especially for $I L$ 6 , in increasing lipid lipolysis, and also in differentiating the thin-tailed sheep breeds from fat-tailed ones.

Finally, validation of three selected DEGs revealed that two of them (i.e. TNNC1 and S100A8) had the same direction of expression in our study and the study of Bakhtiarizadeh et al. (2019). Results of the two studies show that TNNC1 and S100A8 genes had higher and lower expression in fatand thin-tailed sheep breeds, respectively. Troponin C1, slow skeletal muscle and cardiac type (TNNC1) are related with the tenderness of meat (Wang et al., 2020). Also, an important paralog of this gene is troponin $\mathrm{C} 2$, fast skeletal type (TNNC2), which exists in the longissimus muscle area and is strongly related with fat deposition (Silva et al., 2019). S100A8 is a well-known inflammatory-response related gene of obese adipose tissue that emphasize its essential role on the pathogenesis of obesity (Sekimoto et al., 2015). High circulating levels of S100A8 in obese individuals have been confirmed in mice (Sekimoto et al., 2012) and human (Chen et al., 2020). But $S 100 A 8$ gene in two study were up-regulated in Zel (thin-tailed) breed. Unlike the above two genes, the $J U N B$ gene had a different expression direction, and as mentioned above, this gene is enriched in the TNF signaling pathway, which is one of the important pathways for fat lipolysis. In addition, the $J U N B$ gene in the green module of up- regulated genes as a hub gene may play a major role in fat lipolysis.

\section{Conclusions}

In the present study, we compared the gene expression profile of the Ghezel (fat-tailed) with that of the Zel (thin-tailed) sheep breed with focus on genes related to fat metabolism. Our results indicate that known fat metabolism pathways such as "fatty acid metabolism", "fatty acid biosynthesis", "pyruvate metabolism" and "HIF-1 signaling pathway" are more active in the deposition of fat in the tail of Ghezel (fat-tailed) sheep, whereas "TNF signaling pathway" and "MAPK signaling pathway" and related genes stimulate lipolysis in Zel (thin-tailed) sheep. Differential expression of some fat-metabolism-related genes such as $I L-6, N R 4 A I$, SOCS3, ATF3, CREB3L1, HIF-1 $\alpha$, HMGCS1, JUNB, LIPG, NR4A3, FOSL1, VPS35, VPS26A, LTF, LBP and MBOAT2 might contribute to the genetic and morphologic diversity of sheep breeds. IL-6, LIPG and SAAI genes were associated with fat lipolysis and $L T F, L B P, M O G A T 1$ and $M B O A T 2$ were associated with fat deposition at a very high level of expression in the Zel (thin-tailed) and Ghezel (fattailed) breeds, respectively. Strong interaction between the up-regulated DEGs was shown via PPI network analysis. This implies that DEGs of these modules, especially the $I L-6$ gene, are major candidate genes in the tail fat metabolism of sheep. Our findings also showed that enriched modules with significant KEGG pathway terms have a strong association with fat metabolism. In addition, the structural classification of proteins showed that the DEGs under the transcription factor class play an important role in the lipolysis of the Zel 
(thin-tailed) breed. Finally, our findings led to a further understanding of the distribution and regulation of fat deposition in different types of tails in sheep breeds.

Data availability. The generated raw RNA-Seq data were deposited into the NCBI SRA database with BioProject accession number PRJNA598581.

Supplement. The supplement related to this article is available online at: https://doi.org/10.5194/aab-64-53-2021-supplement.

Author contributions. SF carried out the investigation and formal analysis and prepared the original draft. JSG provided resources and helped with the conceptualization and methodology. $\mathrm{KH}$ carried out the formal analysis and took part in writing, reviewing and editing. SAM took part in writing, reviewing and editing. EE took part in writing, reviewing and editing as well as carrying out project administration.

Competing interests. The authors declare that they have no conflict of interest.

Acknowledgements. We cordially acknowledge Mohammad Farhadian and Arash Javanmard for their kind assistance in the analyses and preparation of the paper.

Review statement. This paper was edited by Steffen Maak and reviewed by two anonymous referees.

\section{References}

Albert, R. and Barabási, A.-L.: Statistical mechanics of complex networks, Rev. Mod. Phys., 74, 47, https://doi.org/10.1103/RevModPhys.74.47, 2002.

Andrews, S.: FastQC: a quality control tool for high throughput sequence data, available at: http://www.bioinformatics.babraham. ac.uk/projects/fastqc (last access: 14 February 2021), 2010.

Atti, N., Bocquier, F., and Khaldi, G.: Performance of the fat-tailed Barbarine sheep in its environment: adaptive capacity to alternation of underfeeding and re-feeding periods. A review, Anim. Res., 53, 165-176, 2004.

Auguet, T., Guiu-Jurado, E., Berlanga, A., Terra, X., Martinez, S., Porras, J. A., Ceausu, A., Sabench, F., Hernandez, M., and Aguilar, C.: Downregulation of lipogenesis and fatty acid oxidation in the subcutaneous adipose tissue of morbidly obese women, Obesity, 22, 2032-2038, 2014.

Bakhtiarizadeh, M. R., Salehi, A., Alamouti, A. A., AbdollahiArpanahi, R., and Salami, S. A.: Deep transcriptome analysis using RNA-Seq suggests novel insights into molecular aspects of fat-tail metabolism in sheep, Sci. Rep.-UK, 9, 9203, https://doi.org/10.1038/s41598-019-45665-3, 2019.
Bernard, C., Cassar-Malek, I., Le Cunff, M., Dubroeucq, H., Renand, G., and Hocquette, J.-F.: New indicators of beef sensory quality revealed by expression of specific genes, J. Agr. Food Chem., 55, 5229-5237, 2007.

Bolger, A. M., Lohse, M., and Usadel, B.: Trimmomatic: a flexible trimmer for Illumina sequence data, Bioinformatics, 30, 2114 2120, 2014.

Boulton, T., Dunlop, M., and Court, J.: The growth and development of fat cells in infancy, Pediatr. Res., 12, 908-911, 1978.

Braissant, O., Foufelle, F., Scotto, C., Dauça, M., and Wahli, W.: Differential expression of peroxisome proliferator-activated receptors (PPARs): tissue distribution of PPAR-alpha, -beta, and -gamma in the adult rat, Endocrinology, 137, 354-366, 1996.

Chen, N., Miao, L., Lin, W., Zhou, D.-H., Huang, L., Huang, J., Shi, W.-X., Li, L.-L., Luo, Y.-X., and Liang, H.: Integrated DNA methylation and gene expression analysis identified S100A8 and S100A9 in the pathogenesis of obesity, Research Square, 2, https://doi.org/10.21203/rs.3.rs-68833/v2, 2020.

Chen, S.-H., Chin, C.-H., Wu, H.-H., Ho, C.-W., Ko, M.-T., and Lin, C.-Y.: cyto-Hubba: A Cytoscape plug-in for hub object analysis in network biology, 20th International Conference on Genome Informatics, 2009.

Cheng, X., Zhao, S., Yue, Y., Liu, Z., Li, H., and Wu, J.: Comparative analysis of the liver tissue transcriptomes of Mongolian and Lanzhou fat-tailed sheep, Genet. Mol. Res., 15, e15028572, https://doi.org/10.4238/gmr.15028572, 2016.

Chin, C.-H., Chen, S.-H., Wu, H.-H., Ho, C.-W., Ko, M.-T., and Lin, C.-Y.: cytoHubba: identifying hub objects and subnetworks from complex interactome, BMC Syst. Biol., 8, S11, https://doi.org/10.1186/1752-0509-8-S4-S11, 2014.

Contreras, G. A., Strieder-Barboza, C., De Souza, J., Gandy, J., Mavangira, V., Lock, A. L., and Sordillo, L. M.: Periparturient lipolysis and oxylipid biosynthesis in bovine adipose tissues, PloS One, 12, e0188621, https://doi.org/10.1371/journal.pone.0188621, 2017a.

Contreras, G. A., Strieder-Barboza, C., and Raphael, W.: Adipose tissue lipolysis and remodeling during the transition period of dairy cows, J. Anim. Sci. Biotechno., 8, 41, https://doi.org/10.1186/s40104-017-0174-4, 2017 b.

Damon, M., Denieul, K., Vincent, A., Bonhomme, N., WyszynskaKoko, J., and Lebret, B.: Associations between muscle gene expression pattern and technological and sensory meat traits highlight new biomarkers for pork quality assessment, Meat Sci., 95, 744-754, 2013.

Davidson, A.: The Oxford companion to food, Oxford University Press, Oxford, UK, 2014.

Duszka, K., Bogner-Strauss, J. G., Hackl, H., Rieder, D., Neuhold, C., Prokesch, A., Trajanoski, Z., and Krogsdam, A.-M.: Nr4a1 Is Required for Fasting-Induced Down-Regulation of Ppar $\gamma 2$ in White Adipose Tissue, Mol. Endocrinol., 27, 135-149, 2013.

Ehrlund, A., Mejhert, N., Björk, C., Andersson, R., Kulyté, A., Åström, G., Itoh, M., Kawaji, H., Lassmann, T., and Daub, C. O.: Transcriptional dynamics during human adipogenesis and its link to adipose morphology and distribution, Diabetes, 66, 218 230, https://doi.org/10.2337/db16-0631, 2017.

Ermias, E., Yami, A., and Rege, J.: Fat deposition in tropical sheep as adaptive attribute to periodic feed fluctuation, J. Anim. Breed. Genet., 119, 235-246, 2002. 
Feingold, K. R., Doerrler, W., Dinarello, C. A., Fiers, W., and Grunfeld, C.: Stimulation of lipolysis in cultured fat cells by tumor necrosis factor, interleukin-1, and the interferons is blocked by inhibition of prostaglandin synthesis, Endocrinology, 130, 10 16, 1992.

Fuhrmeister, J., Zota, A., Sijmonsma, T. P., Seibert, O., Cıngır, Ş., Schmidt, K., Vallon, N., de Guia, R. M., Niopek, K., and Diaz, M. B.: Fasting-induced liver GADD $45 \beta$ restrains hepatic fatty acid uptake and improves metabolic health, EMBO Mol. Med., 8, 654-669, 2016.

Gokdal, O., Ulker, H., Karakus, F., Cengiz, F., Temur, C., and Handil, H.: Growth, feedlot performance and carcass characteristics of Karakas and crossbred lambs (F1)(Ile de France $\times$ Akkaraman $(\mathrm{G} 1) \times$ Karakas) under rural farm conditions in Turkey, S. Afr. J. Anim. Sci., 34, 223-232, 2004.

Green, A., Dobias, S. B., Walters, D., and Brasier, A. R.: Tumor necrosis factor increases the rate of lipolysis in primary cultures of adipocytes without altering levels of hormone-sensitive lipase, Endocrinology, 134, 2581-2588, 1994.

Grisouard, J., Bouillet, E., Timper, K., Radimerski, T., Dembinski, K., Frey, D. M., Peterli, R., Zulewski, H., Keller, U., and Müller, B.: Both inflammatory and classical lipolytic pathways are involved in lipopolysaccharide-induced lipolysis in human adipocytes, Innate Immun., 18, 25-34, 2012.

Han, J., Meng, Q., Shen, L., and Wu, G.: Interleukin-6 induces fat loss in cancer cachexia by promoting white adipose tissue lipolysis and browning, Lipids Healt Dis., 17, 14, https://doi.org/10.1186/s12944-018-0657-0, 2018.

Hauner, H., Petruschke, T., Russ, M., Röhrig, K., and Eckel, J.: Effects of tumour necrosis factor alpha (TNF $\alpha)$ on glucose transport and lipid metabolism of newly-differentiated human fat cells in cell culture, Diabetologia, 38, 764-771, 1995.

Justine, E. Y., Han, S.-Y., Wolfson, B., and Zhou, Q.: The role of endothelial lipase in lipid metabolism, inflammation, and cancer, Histol. Histopathol., 33, 1-10, https://doi.org/10.14670/HH-11905, 2018

Kamalzadeh, A., Rajabbaigy, M., and Kiasat, A.: Livestock production systems and trends in livestock industry in Iran, J. Agri. Soc. Sci., 4, 183-188, 2008.

Kashan, N., Azar, G. M., Afzalzadeh, A., and Salehi, A.: Growth performance and carcass quality of fattening lambs from fattailed and tailed sheep breeds, Small Ruminant Res., 60, 267271, 2005

Kawakami, M., Murase, T., Ogawa, H., Ishibashi, S., Mori, N., Takaku, F., and Shibata, S.: Human recombinant TNF suppresses lipoprotein lipase activity and stimulates lipolysis in 3T3-L1 cells, J. Biochem., 101, 331-338, 1987.

Kim, J. H., Qu, A., Reddy, J. K., Gao, B., and Gonzalez, F. J.: Hepatic oxidative stress activates the Gadd45b gene by way of degradation of the transcriptional repressor STAT3, Hepatology, 59, 695-704, https://doi.org/10.1002/hep.26683, 2014.

Langmead, B. and Salzberg, S. L.: Fast gapped-read alignment with Bowtie 2, Nat. Methods, 9, 357-359, https://doi.org/10.1038/nmeth.1923, 2012.

Le, V.-H. and Kim, S.-R.: K-strings algorithm, a new approach based on Kmeans, Proceedings of the 2015 Conference on research in adaptive and convergent systems, New York, NY, United States, 15-20, https://doi.org/10.1145/2811411.2811472, 2015.
Li, B., Qiao, L., An, L., Wang, W., Liu, J., Ren, Y., Pan, Y., Jing, J., and Liu, W.: Transcriptome analysis of adipose tissues from two fat-tailed sheep breeds reveals key genes involved in fat deposition, BMC Genomics, 19, 338, https://doi.org/10.1186/s12864018-4747-1, 2018.

Li, H., Handsaker, B., Wysoker, A., Fennell, T., Ruan, J., Homer, N., Marth, G., Abecasis, G., and Durbin, R.: The sequence alignment/map format and SAMtools, Bioinformatics, 25, 20782079, 2009.

Liss, K. H., Lutkewitte, A. J., Pietka, T., Finck, B. N., Franczyk, M., Yoshino, J., Klein, S., and Hall, A. M.: Metabolic importance of adipose tissue monoacylglycerol acyltransferase 1 in mice and humans, J. Lipid Res., 59, 1630-1639, 2018.

Luo, B., Zou, T., Lu, N., Chai, F., Ye, X., Wang, Y., and Qi, Y.: Role of suppressor of cytokine signaling 3 in lipid metabolism: Analysis based on a phage-display human liver cDNA library, Biochem. Bioph. Res. Co., 416, 39-44, 2011.

Ma, L., Zhang, M., Jin, Y., Erdenee, S., Hu, L., Chen, H., Cai, Y., and Lan, X.: Comparative transcriptome profiling of mRNA and lncRNA related to tail adipose tissues of sheep, Frontiers in Genetics, 9, 365, https://doi.org/10.3389/fgene.2018.00365, 2018.

Ma, Y., Gao, M., Sun, H., and Liu, D.: Interleukin-6 gene transfer reverses body weight gain and fatty liver in obese mice, Bba.Mol. Basis Dis., 1852, 1001-1011, 2015.

Matthews, V. B., Allen, T., Risis, S., Chan, M., Henstridge, D. C., Watson, N., Zaffino, L., Babb, J. R., Boon, J., and Meikle, P. J.: Interleukin-6-deficient mice develop hepatic inflammation and systemic insulin resistance, Diabetologia, 53, 2431-2441, 2010.

Miao, X. and Luo, Q.: Genome-wide transcriptome analysis between small-tail Han sheep and the Surabaya fur sheep using high-throughput RNA sequencing, Reproduction, Reproduction, 145, 587-596, https://doi.org/10.1530/REP-12-0507, 2013.

Moradi, M. H., Nejati-Javaremi, A., Moradi-Shahrbabak, M., Dodds, K. G., and McEwan, J. C.: Genomic scan of selective sweeps in thin and fat tail sheep breeds for identifying of candidate regions associated with fat deposition, BMC Genet., 13, 10, https://doi.org/10.1186/1471-2156-13-10, 2012.

Moreno-Navarrete, J. M., Jové, M., Padró, T., Boada, J., Ortega, F., Ricart, W., Pamplona, R., Badimón, L., Portero-Otín, M. and Fernández-Real, J. M.: Adipocyte lipopolysaccharide binding protein (LBP) is linked to a specific lipidomic signature, Obesity, 25, 391-400, 2017.

Mortazavi, A., Williams, B. A., McCue, K., Schaeffer, L., and Wold, B.: Mapping and quantifying mammalian transcriptomes by RNA-Seq, Nat. Methods, 5, 621-628, https://doi.org/10.1038/nmeth.1226, 2008.

Nabavi, R., Alijani, S., Taghizadeh, A., Rafat, S. A., and Bohlouli, M.: Genetic study of reproductive traits in Iranian native Ghezel sheep using Bayesian approach, Small Ruminant Res., 120, 189195, 2014.

Nejati-Javaremi, A., Izadi, F., Rahmati, G., Moradi, M., and Izadi, F.: Selection in fat-tailed sheep based on two traits of fat-tail and body weight versus single-trait total body weight, Int. J. Agric. Biol., 9, 645-648, 2007.

Nonogaki, K., Fuller, G. M., Fuentes, N. L., Moser, A. H., Staprans, I., Grunfeld, C., and Feingold, K. R.: Interleukin-6 stimulates hepatic triglyceride secretion in rats, Endocrinology, 136, 2143 2149, 1995. 
Pourlis, A. F.: A review of morphological characteristics relating to the production and reproduction of fat-tailed sheep breeds, Trop. Anim. Health Pro., 43, 1267-1287, 2011.

Queipo-Ortuño, M. I., Escoté, X., Ceperuelo-Mallafré, V., GarridoSanchez, L., Miranda, M., Clemente-Postigo, M., Pérez-Pérez, R., Peral, B., Cardona, F., and Fernández-Real, J. M.: FABP4 dynamics in obesity: discrepancies in adipose tissue and liver expression regarding circulating plasma levels, PloS One, 7, e4860, https://doi.org/10.1371/journal.pone.0048605, 2012.

Ruderman, N. B., Keller, C., Richard, A.-M., Saha, A. K., Luo, Z., Xiang, X., Giralt, M., Ritov, V. B., Menshikova, E. V., and Kelley, D. E.: Interleukin-6 regulation of AMP-activated protein kinase: potential role in the systemic response to exercise and prevention of the metabolic syndrome, Diabetes, 55, S48-S54, https://doi.org/10.2337/db06-S007, 2006.

Sekimoto, R., Kishida, K., Nakatsuji, H., Nakagawa, T., Funahashi, T., and Shimomura, I.: High circulating levels of S100A8/A9 complex (calprotectin) in male Japanese with abdominal adiposity and dysregulated expression of S100A8 and S100A9 in adipose tissues of obese mice, Biochem. Bioph. Res. Co., 419, 782789, 2012

Sekimoto, R., Fukuda, S., Maeda, N., Tsushima, Y., Matsuda, K., Mori, T., Nakatsuji, H., Nishizawa, H., Kishida, K., and Kikuta, J.: Visualized macrophage dynamics and significance of S100A8 in obese fat, P. Natl. Acad. Sci. USA, 112, E2058-E2066, 2015.

Shi, L., Liu, L., Lv, X., Ma, Z., Yang, Y., Li, Y., Zhao, F., Sun, D., and Han, B.: Polymorphisms and genetic effects of PRLR, MOGAT1, MINPP1 and CHUK genes on milk fatty acid traits in Chinese Holstein, BMC Genet., 20, 1-8, 2019.

Silva, R. P., Berton, M. P., Grigoletto, L., Carvalho, F. E., Silva, R. M., Peripolli, E., Castro, L. M., Ferraz, J. B. S., Eler, J. P., and Lôbo, R. B.: Genomic regions and enrichment analyses associated with carcass composition indicator traits in Nellore cattle, J. Anim. Breed. Genet., 136, 118-133, 2019.

Symonds, M. E.: Brown adipose tissue growth and development, Scientifica, 2013, 305763, https://doi.org/10.1155/2013/305763, 2013.

Symonds, M. E., Pope, M., Sharkey, D., and Budge, H.: Adipose tissue and fetal programming, Diabetologia, 55, 1597-1606, 2012.

Szklarczyk, D., Morris, J. H., Cook, H., Kuhn, M., Wyder, S., Simonovic, M., Santos, A., Doncheva, N. T., Roth, A., and Bork, P.: The STRING database in 2017: quality-controlled proteinprotein association networks, made broadly accessible, Nucleic Acids Res., 45, 362-368, https://doi.org/10.1093/nar/gkw937, 2016.

Trapnell, C., Pachter, L., and Salzberg, S. L.: TopHat: discovering splice junctions with RNA-Seq, Bioinformatics, 25, 1105-1111, 2009.

Trapnell, C., Roberts, A., Goff, L., Pertea, G., Kim, D., Kelley, D. R., Pimentel, H., Salzberg, S. L., Rinn, J. L., and Pachter, L.: Differential gene and transcript expression analysis of RNA-seq experiments with TopHat and Cufflinks, Nat. Protoc., 7, 562578, https://doi.org/10.1038/nprot.2012.016, 2012.

Valizadeh, R.: Iranian sheep and goat industry at a glance, Stress Management in Small Ruminant Production and Product Processing, Jaipur, India, 2010.

van Hall, G., Steensberg, A., Sacchetti, M., Fischer, C., Keller, C., Schjerling, P., Hiscock, N., Møller, K., Saltin, B., and Febbraio,
M. A.: Interleukin-6 stimulates lipolysis and fat oxidation in humans, J. Clin. Endocr. Metab., 88, 3005-3010, 2003.

Vatankhah, M., Moradi-Sharbabak, M., Nejati-Javaremi, A., Miraei-Ashtiani, S., and Vaez-Torshizi, R.: A study of external fat-tail dimensions and their relationships with fat-tail weight in lori-bakhtiari breed of sheep, Journal of Science and Technology of Agriculture and Natural Resources, 10, 445-546, 2006.

Walton, R. G., Zhu, X., Tian, L., Heywood, E. B., Liu, J., Hill, H. S., Liu, J., Bruemmer, D., Yang, Q., and Fu, Y.: AP2-NR4A3 transgenic mice display reduced serum epinephrine because of increased catecholamine catabolism in adipose tissue, Am. J. Physiol.-Endoc. M., 311, E69-E81, 2016.

Wang, M., Zhao, S., and Tan, M.: bZIP transmembrane transcription factor CREBH: Potential role in non-alcoholic fatty liver disease, Mol. Med. Rep., 13, 1455-1462, https://doi.org/10.3892/mmr.2015.4749, 2016.

Wang, S., Raza, S. H. A., Mei, C., Zhu, K., Garcia, M., Schreurs, N. M., Liang, C., Yang, X., and Zan, L.: Transcriptome profiling reveals differential expression of genes potentially involved in muscle and adipose tissue development of cattle, Electron. J. Biotechn., 48, 72-77, 2020.

Wang, X., Zhou, G., Xu, X., Geng, R., Zhou, J., Yang, Y., Yang, Z., and Chen, Y.: Transcriptome profile analysis of adipose tissues from fat and short-tailed sheep, Gene, 549, 252-257, 2014.

Wang, Y. C., Kuo, W. H., Chen, C. Y., Lin, H. Y., Wu, H. T., Liu, B. H., Chen, C. H., Mersmann, H. J., Chang, K. J., and Ding, S. T.: Docosahexaenoic acid regulates serum amyloid A protein to promote lipolysis through down regulation of perilipin, J. Nutr. Biochem., 21, 317-324, 2010.

Wickramasinghe, S., Cánovas, A., Rincón, G., and Medrano, J. F.: RNA-sequencing: a tool to explore new frontiers in animal genetics, Livest. Sci., 166, 206-216, 2014.

Wu, Y., Song, P., Zhang, W., Liu, J., Dai, X., Liu, Z., Lu, Q., Ouyang, C., Xie, Z., and Zhao, Z.: Activation of AMPK $\alpha 2$ in adipocytes is essential for nicotine-induced insulin resistance in vivo, Nat. Med., 21, 373-382, https://doi.org/10.1038/nm.3826, 2015.

Xu, M., Joo, H.-J., and Paik, Y.-K.: Novel functions of lipid-binding protein 5 in Caenorhabditis elegans fat metabolism, J. Biol Chem., 286, 28111-28118, 2011.

$\mathrm{Xu}$, Y., Zhang, Y., and Ye, J.: IL-6: A potential role in cardiac metabolic homeostasis, Int. J. Mol. Sci., 19, 2474, https://doi.org/10.3390/ijms19092474, 2018.

Yan, H., Kermouni, A., Abdel-Hafez, M., and Lau, D. C.: Role of cyclooxygenases COX-1 and COX-2 in modulating adipogenesis in 3T3-L1 cells, J. Lipid Res., 44, 424-429, 2003.

Yang, X., Zhang, X., Heckmann, B. L., Lu, X., and Liu, J.: Relative contribution of adipose triglyceride lipase and hormone-sensitive lipase to tumor necrosis factor- $\alpha$ (TNF- $\alpha$ )-induced lipolysis in adipocytes, J. Biol. Chem., 286, 40477-40485, 2011.

Zhang, W., Mottillo, E. P., Zhao, J., Gartung, A., VanHecke, G. C., Lee, J.-F., Maddipati, K. R., Xu, H., Ahn, Y.-H., and Proia, R. L.: Adipocyte lipolysis-stimulated interleukin-6 production requires sphingosine kinase 1 activity, J. Biol. Chem., 289, 32178-32185, 2014.

Zhao, N., Li, X., Feng, Y., Han, J., Feng, Z., Li, X., and Wen, Y.: The nuclear orphan receptor Nur77 alleviates palmitate-induced fat accumulation by down-regulating G0S2 in HepG2 Cells, Sci 
Rep.-UK, 8, 4809, https://doi.org/10.1038/s41598-018-23141-8, 2018.

Zheng, Y., Blair, D., and Bradley, J. E.: Phyletic distribution of fatty acid-binding protein genes, PloS One, 8, e77636, https://doi.org/10.1371/journal.pone.0077636, 2013.
Zhou, G., Wang, X., Yuan, C., Kang, D., Xu, X., Zhou, J., Geng, R., Yang, Y., Yang, Z., and Chen, Y.: Integrating miRNA and mRNA expression profiling uncovers miRNAs underlying fat deposition in sheep, BioMed Res. Int., 17, 1857580, https://doi.org/10.1155/2017/1857580, 2017. 